\title{
Elevated circulating endothelin-1 as a potential biomarker for high- risk COVID-19 severity
}

\author{
Z. Shadi Farhangrazi ${ }^{1,2}$ and S. Moein Moghimi ${ }^{3 *}$ \\ ${ }^{I}$ S. M. Discovery Group Inc., Colorado, USA \\ ${ }^{2}$ S. M. Discovery Group Ltd., Durham, UK \\ ${ }^{3}$ School of Pharmacy, Newcastle University, Newcastle upon Tyne NE $17 R U$, UK
}

Submitted: June 3, 2020

Accepted: June 16, 2020

Published: June 29, 2020

\section{Graphical Abstract}

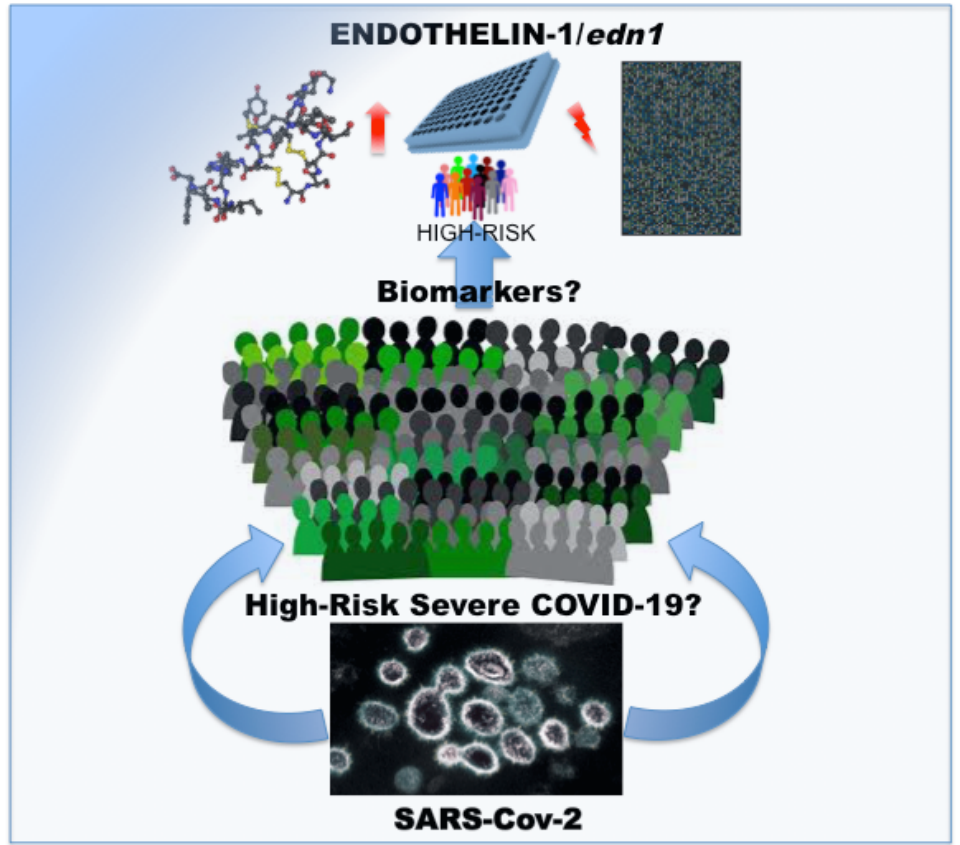

\section{Abstract}

There is a disproportionately higher rate of adverse outcomes in coronavirus disease 2019 (COVID19) patients of the male sex, select ethnicities, and individuals with obesity and those that tend to have endothelial dysfunction (e.g., hypertensives, diabetics and individuals with coronary heart disease and respiratory system diseases). Endotheliitis across vascular beds of multiple organs, thrombosis and ischaemia, are among common pathological features of severe COVID-19 cases. Endothelin-1 (ET-1) is the most potent vasoconstrictor of the human cardiovascular system and a culprit of endothelial dysfunction. Elevated circulating ET-1 levels are a predictor of cardiovascular disease status and have been correlated with racial/ethnic differences in microvascular and macrovascular disease severity and prognosis as well as with older age-associated endothelial dysfunction. Here, we propose elevated circulating ET-1 levels as a plausible biomarker and prognostic tool in predicting individuals at high risk of developing severe COVID-19. In addition to this, we also propose ET-1 gene variants and expression patterns might also cause a predisposition for an increased risk of severe acute respiratory syndrome coronavirus- 2 infection severity in some individuals and/or populations.

\section{Keywords:}

Biomarkers; COVID-19; Endothelin-1; Endothelin-1 gene; NF-кB; SARS-CoV-2

\section{*Correspondence: S. Moein Moghimi, seyed.moghimi@ncl.ac.uk, Z. Shadi Farhangrazi;}




\section{Purpose and Rationale}

Precision medicine is an emerging approach to disease management, which utilises data from genomics, environment and lifestyle to predict more accurate prevention and treatment strategies for a particular disease in different populations. The emergence of precision medicine is, therefore, helping with the discovery of novel drug targets, drug repurposing/repositioning, nanomedicine engineering, development of nanotechnologybased companion diagnostics, and improving clinical trial outcomes. The coronavirus disease 2019 (COVID-19), which is caused by severe acute respiratory syndrome coronavirus-2 (SARS-CoV-2), has created a pandemic across the world with a disproportionately higher risk of adverse outcomes in some patients and populations. Precision medicine offers an opportunity to identify robust biomarkers that could predict individuals at higher risk of severe COVID-19 and to combat the pandemic. Such progress could trigger the potential development of robust and precise multifunctional nanotechnology-based diagnostics such as multiplex plasmonic platforms for simultaneous detection of the virus and risk biomarkers. Here, based on the available evidence of SARS-CoV-2 interaction with endothelium, the renin-angiotensin-aldosterone system (RAAS) and endothelin system, and the resultant disease manifestations [1-3], we propose endothelin-1 (ET-1) as a plausible robust biomarker and prognostic tool in predicting individuals at high risk of developing severe COVID-19.

\section{Introduction and Discussion}

\section{Endothelial dysfunction}

The vascular endothelium plays an essential role in the regulation of vascular tone and the maintenance of vascular homeostasis through secretions of potent vasorelaxant (e.g., nitric oxide) and vasoconstricting (e.g., ET-1) substances [1]. Endothelial dysfunction is a hallmark of various human disease states and is characterised by diminished production/bioavailability of nitric oxide presumably as a result of suppressed endothelial nitric oxide synthase, and/or an increase in endotheliumderived contracting factors (notably ET-1), which shifts the vascular equilibrium towards more vasoconstriction [1]. ET-1 is not only the most potent vasoconstrictor in the human cardiovascular system but also its complex and multifaceted interactions with the RAAS are well documented [2,3]. ET-1 plays multiple roles in cardiovascular, pulmonary, in renal and neural physiology, and as such, differential and tissue-specific production of ET-1 is tightly regulated. Biological factors and mediators such as angiotensin II, cytokines and free radicals promote ET-1 secretion, whereas nitric oxide, prostacyclin and cyclic GMP reduce ET1 release [2,3]. The critical role of ET-1 in pathophysiological changes associated with both microvascular and macrovascular diseases are well known and include proinflammatory, proliferative and procoagulatory states [2].

Increased circulating ET-1 levels have been found in smokers and those with conditions such as hypertension, atherosclerosis, coronary heart disease, cerebrovascular diseases, diabetes and sepsis [2]. Elevated circulating ET-1 levels have further been correlated with population differences in disease severity and prognosis as well as older age [2,4-11]. For instance, there are higher rates of hypertension among African American and Hispanic persons than in non-Hispanic white persons [4]. As such, ET-1 has been implicated as a key mediator in the pathogenesis of hypertension and vascular disease among African Americans, as African Americans with systemic hypertension have higher circulating levels of both ET-1 and ET-1 precursors than white people with hypertension $[5,6]$. The aetiology of racial differences in the prevalence and severity of hypertension has further been noted in other parts of the world. For example, plasma concentrations of ET-1 were shown to be significantly higher in hypertensive Gulf Arab patients compared with white hypertensives [7]. Furthermore, the higher levels of ET-1 have predicted disease severity and mortality in many forms of pulmonary arterial hypertension, including in HIV infection [8]. Alterations in the ET-1 gene $(e d n 1)$ expression patterns have also been documented in the pathogenesis and progression of various human diseases [12]. For instance, genetic polymorphisms such as a common adenine insertion in the 5'untranslated region of $e d n 1$ results in elevated mRNA levels, and this has been associated with 
essential hypertension [13]. Also, edn1 variants leading to high circulating ET-1 levels have been identified as a risk factor for coronary artery disease and intracerebral haemorrhage in certain populations including a Chinese Han population $[9,10]$. Endothelial function also deteriorates with aging and plasma ET-1 concentration significantly increases with ageing, but exercise reduces plasma ET-1 level in older individuals [11].

Can circulating ET-1 levels serve as a predictive biomarker for COVID-19 severity?

Since the COVID-19 outbreak, we have witnessed a disproportionately higher rate of adverse outcomes in patients with pre-existing endothelial dysfunction, notably in those with hypertension, coronary heart disease, respiratory system diseases, diabetes, obesity and of the male sex $[14,15]$. Vascular and thrombotic complications, including symptomatic acute pulmonary embolism, deepvein thrombosis, ischaemic stroke, myocardial infarction and systemic arterial embolism have been reported in severe COVID-19 cases $[15,16]$. These complications are presumably related to endothelial cell infection with SARSCoV-2 through its cognate host angiotensinconverting enzyme 2 (ACE2) receptor, which is highly expressed by arterial and venous endothelial cells [17], resulting in endotheliitis, thromboembolism and ischaemia. The overall COVID-19 severity in infants and children is significantly milder than in adults $[18,19]$, but in rare cases, some children have developed an inflammatory, multisystem syndrome similar to Kawasaki disease [20,21]. Older age and race/ethnicity have further been identified as risk factors in severe COVID-19 cases and deaths [22]. For instance, the Centers for Disease Control and Prevention report a disproportionate burden of illness and death rates among Black/African Americans and Hispanic/Latino persons than white or Asian persons [23]. Some reports have attributed these to differences in socioeconomic status levels and living conditions, combined with restricted access to healthcare services [24,25]. However, by considering the existing correlations between ET-1 and endothelial dysfunction, including in Kawasaki disease [26], we propose that elevated circulating ET-1 levels might also serve as a prominent biomarker and prognostic tool to identify individuals with the greatest risks of severe COVID-19. This suggestion might, therefore, explain the disparities seen in COVID-19 severity and patients with cardiometabolic comorbidities. For instance, nitric oxide deficiency in endothelial dysfunction may contribute to viral cellular entry and subsequent proinflammatory reactions, since nitric oxide apparently interferes with the interaction between coronavirus S-protein and ACE2 [27]. Furthermore, genetic differences such as ET-1 gene variants leading to high levels of circulating ET-1 and/or ET-1 receptor A and B type gene variants might also cause a predisposition for an increased risk of SARS$\mathrm{CoV}-2$ infection severity and endotheliitis in some individuals and/or populations.

The rate of transcription from edn1 primarily controls ET-1 bioavailability [12], and alterations in cellular redox states are known to modify DNA binding and transactivation activities of many transcriptional activators [28]. The edn1 promoter contains both common cis-acting elements and cell-type-specific and inducible elements. Many hormones (e.g., steroid hormones such as aldosterone), stimuli (e.g., hypoxia) and cell signals (e.g., the redoxsensitive transcriptional factor NF- $\mathrm{kB}$ ) regulate edn1 gene transcription through cis-acting elements in the edn1 promoter [12]. The role of $\mathrm{NF}-\kappa \mathrm{B}$ is of particular interest due to the observed cytokine storm and hyper inflammation in severe COVID-19 cases [29]. Recently, dexamethasone, which reduces NF$\kappa \mathrm{B}$-mediated transcriptional activity without altering I $\mathrm{I} B$ protein levels or the nuclear translocation of NF- $\kappa \mathrm{B}$, has shown efficacy in treating critically ill COVID-19 patients. The $e d n 1$ promoter contains three functional NF- $\mathrm{KB}$ binding sites and many cytokines such as tumour necrosis factor- $\alpha$, interferon- $\gamma$ and interleukin- $1 \beta$ are capable of activating NF- $\kappa \mathrm{B}-$ dependent edn1 expression [12]. Glucose has also been shown to stimulate the recruitment of $\mathrm{NF}-\kappa \mathrm{B}$ as well as histone acetyltransferase p300 to the edn1 promoter, leading to an increase in histone $\mathrm{H} 3$ acetylation [30]. Moreover, the edn1 promoter also contains a consensus-binding motif for the vascular endothelial zinc factor 1 (Vezf1), which is 
exclusively expressed in vascular endothelial and haematopoietic cells [31]. Rac1-specific GTPase p68RacGAP has been identified as a regulatory cell signal for Vezf1 and coexpression of Vezf1 and p68RacGAP in endothelial cells inhibits Vezf1 transcriptional activation of the $e d n 1$ gene in a dose-dependent manner [32]. It also appears that a Vezf1 binding site in the edn 1 promoter is involved in both basal and insulin-stimulated increases in edn1 expression in endothelial cells [33]. The proposed mechanism involves phosphatidylinositol-3 kinase-dependent inhibition of the glycogen synthases kinase $3 \beta$ leading to depression of Vezf1 [33]. Intriguingly, a recent study has suggested that SARS-CoV-2 may cause pleiotropic alterations of glucose metabolism, leading to new-onset diabetes in COVID-19 patients who seemingly were healthy before the infection [34]. Again, these observations may partly be related to glucose and insulin-mediated increase in edn 1 expression, which in turn may be dependent on corresponding edn1 promoter polymorphisms. In line with this hypothesis, another important response element in the ednl promoter is the transcriptional factor activator protein-1 (AP-1) binding site [12]. AP-1 is also known to mediate genomic responses to many proinflammatory and proliferative signals. For instance, protein kinase $\mathrm{C}$-dependent activation of AP-1 is an important mechanism for edn1 stimuli such as thrombin and angiotensin II $[35,36]$. Since angiotensin II is hydrolysed to angiotensin-1-7 by ACE2 [1,3], dysregulation of ACE2 in SARS-CoV-2 may facilitate lung injury [37]. Thus, angiotensin II-mediated AP1 activation and recruitment to the edn1 promoter resulting in ET-1 overproduction may be a plausible mechanism contributing to the lung injury. This suggestion is consistent with the observation from a small study showing elevated levels of plasma angiotensin II in COVID-19 patients, which further correlated with both total viral load and the extent of lung injury [38]. We further emphasise that in response to hypoxia, the edn 1 promoter recruits AP-1 as well as other factors such as p300, hypoxia-inducible factor 1 , and the transcriptional factor GATA-2 [39].

Finally, many other emerging regulatory mechanisms that can alter $e d n 1$ transcription in a tissue-specific manner and include intracellular calcium and calmodulin levels, mechanotransduction signals and extracellular hypertonicity [12], but their roles within the context of COVID-19 severity need detailed assessment. Epigenetic factors must further be considered since tissue-specific ednl expression is also modulated by DNA methylation [12].

\section{Conclusions}

Here, we present a hypothesis suggesting that elevated circulating ET-1 levels might serve as a plausible biomarker for identifying individuals with the greatest risks of severe COVID-19. Immunoreactive ET-1 is routinely measured by both radioimmunoassay and enzyme immunometric assay [40]. However, plasma concentrations of ET-1 are in the low picomolar range making direct measurements in the plasma difficult [40]. Accordingly, endothelin is extracted from plasma and concentrated before measurement, but extraction procedures and recovery conditions can dramatically affect intra- and inter-assay variability [40]. However, recent developments in plasmonic gold chips with enhanced near-infrared fluorescence [41] could be adopted for ET-1 detection in plasma without prior extraction, since plasmonic platforms offer detection with up to several orders of magnitude higher sensitivity than with methods such as the conventional enzyme-linked immunosorbent assay. Finally, while the validity of ET-1 hypothesis is to be confirmed, concerted genetic testing of SARS-CoV-2 infected individuals is expected to reveal population genetic heterogeneity and related polymorphisms leading to the identification of broader biomarkers providing a greater understanding of the differences and disparities in COVID-19 outcomes and severity. These may include genes encoding the NF- $\mathrm{BB}$ DNA binding subunit (NFKB1) [42] and STING (stimulator of interferon genes, encoded by TMEM173) [43]. Collectively, these efforts could select and prioritise individuals for vaccination as well as improve clinical outcomes through patient stratification with appropriate drug combinations. 


\section{Conflict of interest}

The authors declare no competing interests. For signed statements, please contact the journal office: editor@precisionnanomedicine.com

Quote this article as Farhangrazi ZS, Moghimi SM, Elevated circulating endothelin-1 as a potential biomarker for high-risk COVID-19 severity, Precis. Nanomed. 2020;3(2):622-628, https://doi.org/10.33218/001c.13525

\section{References}

1. D. S. Celermajer, Endothelial dysfunction: does it matter? Is it reversible? J. Am. Coll. Cardiol. 30, 325-333, 1997.

2. N. Dhaun, and D. J. Webb, Endothelins in cardiovascular biology and therapeutics, Nat. Rev. Cardiol. 16, 491-502, 2019.

3. P. G. Rossi, A. Sacchetto, M. Cesari, and A. C. Pessina, Interactions between endothelin-1 and the renin-angiotensin-aldosterone system, Cardiovasc. Res. 43, 300-307, 1999.

4. N. Al-Naamani, J. K. Paulus, K. E. Roberts, M. W. Pauciulo, K. Lutz, and W. C. Nichols, Racial and ethnic differences in pulmonary arterial hypertension, Pulm. Cir. 7, 793-796, 2017.

5. S. Ergul, D. C. Parish, D. Puett, and A. Ergul, Racial differences in plasma endothelin-1 concentrations in individuals with essential hypertension, Hypertension 28, 652-655, 1996.

6. M. D. Jankowich, W-C. Wu, and G. Choudhary, Association of elevated plasma endothelin-1 levels with pulmonary hypertension, mortality, and heart failure in African American individuals, JAMA Cardiol. 1, 461-469, 2016.

7. E. Obineche, A. M. Abdulle, A. M. Bokhari, J. Y. Yasin, and M. P. T. Gillett, Circulating levels of endothelin-1 in a homogenous Gulf Arab population with untreated essential hypertension, Ann. Saud. Med. 26, 364-369, 2006.

8. R. V. Parikh, Y. Ma, R. Scherzer, A. S. Heringer, J. S. Macgregor, J. N. Martin, S. G. Deeks, P. Ganz, and P. Y. Hsue, Endothelin-1 predicts hemodynamically assessed pulmonary arterial hypertension in HIV infection, PLoS One 11, e0146355, 2016.

9. W. Zhang, W. Zhao, C. Ge, X. Li, X. Yang, Y. Xiang, and Z. Sun, Genetic relationship between endothelin-1 gene polymorphisms and intracerebral haemorrhage among Chinese Han people, Med. Sci. Monit. 26, 919110-6, 2020.

10. L-L. Liang, L. Chen, M-Y. Zhou, M-Y. Cai, J. Cheng, Y., Chen, S-K. You, L-B. Chen, Z-B. Tang, $\mathrm{X}-\mathrm{L}$. Yang, et al., Genetic susceptibility of five tagSNPs in the endothelin-1 (EDN1) gene to coronary artery disease in a Chinese Han population, Biosci. Rep. 38, BSR20171320, 2018.

11. S. Maeda, T. Tanabe, T. Miyauchi, T. Otsuki, J. Sugawara, M. Lemitsu, S. Kuna, R. Ajisaka, I. Yamaguchi, and M. Matsuda, Aerobic exercise training reduces plasma endothelin-1 concentration in older woman, J. Appl. Physiol. 95, 336-341, 2003.

12. L. R. Stow, M. E. Jacobs, C. S. Wingo, and B. D. Cain, Endothelin-1 gene regulation, FASEB J. $25,16-28,2011$.

13. K. Popowski, B. Sperker, H. K. Kroemer, U. John, M. Laule, K. Stangl, and I. Cascorbi, Functional significance of a hereditary adenine insertion variant in the 5'-UTR of the endothelin-1 gene, Pharmacogenetics 13, 445-451, 2003.

14. Z. Wu, and J. M. McGoogan, Summary of a report of 72314 cases from the Chinese Center for Disease Control and Prevention, JAMA 323, 1239-1242, 2020.

15. Z. Varga, A. J. Flammer, P. Steiger, M. Haberceker, R. Andermatt, A. S. Zinkernagel, M. R. Mehra, R. A. Schuepbach, F. Ruschitzka, and H. Moch, Endothelial cell infection and endotheliitis in COVID-19, Lancet 395, 1417-1418, 2020.

16. F. A. Klok, M. J. H. A. Kruip, N. J. M. van der Meer, M. S. Arbous, D. A. M. P. J. Gommers, K. M. Kant, F. H. J. Kaptein, J. van Passen, M. A. M. Stals, M. V. Huisman, and H. Endeman, Incidence of thrombotic complications in critically ill ICU patients with COVID-19, Thromb. Res. 191, 145-147, 2020. 
17. Hamming, W. Timens, M. L. C. Bulthuis, A. T. Lely, G. J. Navis, and H. van Goor, Tissue distribution of ACE2 protein, the functional receptor for SARS coronavirus. A first step in understanding SARS pathogenesis, J. Pathol. 203, 631-637, 2004.

18. CDC COVID-19 Response Team (2020). Coronavirus disease 2019 in children-United States, February 12-April 2, 2020, MMWR Morb. Mortal. Wkly. Rep. 69, 422-426, 2020.

19. L. S. Shekerdemian, N. R. Mahmood, K. K. Wolfe, B. J. Riggs, C. E. Ross, C. A. McKiernan, S. M. Heidemann, L. C. Kleinman, A. I. Sen, M. W. Hall, et al., Characteristics and outcomes of children with coronavirus disease 2019 (COVID-19) infection admitted to US and Candaian pediatric intensive care units, JAMA Pediat. doi: 10.1001/jamapediatrics.2020.1948, 2020.

20. E. Whittaker, A. Bamford, J. Kenny, M. Kaforou, C. E. Jones, P. Shah, P. Ramnarayan, A. Fraisse, O. Miller, P. Davies, et al., Clinical characteristics of 58 children with a pediatric inflammatory multisystem syndrome temporally associated with SARS-CoV-2, JAMA, e2010369, doi: 10.1001/jama.2020.10369, 2020.

21. L. Verdoni, A. Mazza, A. Gervasoni, L. Martelli, M. Ruggeri, M. Ciuffreda, E. Bonanomi, and L. D'Antiga, An outbreak of severe Kawasaki-like disease at the Italian epicentre of the SARS-CoV2 epidimic: an observational cohort study, Lancet 395, 1771-1778, 2020.

22. M. Webb Hooper, A. M. Nápoles, and E. J. Pérez-Stable, COVID-19 and racial/ethnic disparities, JAMA doi: 10.1001/jama.2020.8598, 2020.

23. S. Garg, L. Kim, M. Whitaker, A. O’Halloran, C. Cummings, R. Holstein, M. Prill, S. J. Chai, P. D. Kirley, N. B. Alden, et al., Hospitalisation rates and characteristics of patients hospitalised with laboratory-confirmed coronavirus disease 2019 - COVID-NET, 14 States, March 1-30, 2020, MMWR Morb. Mort. Wkly. Rep. 69, 458-464, 2020.

24. G. Anderson, J. W. Frank, C. D. Naylor, W. Wodchis, and P. Feng, Using socioeconomics to counter disparities arising from the covid-19 pandemic, BMJ 369, m2149, 2020.

25. M. Stafford, and S. Deeny, Inequalities and deaths involving COVID-19, What the links between inequalities tell us, https://www.health.org.uk/news-and-comment/blogs/inequalities-and-deathsinvolving-covid-19, 2020.

26. T. Morise, Y. Takeuchi, R. Takeda, U. Karayalcin, A. Yachie, and T. Miyawaki, Increased plasma endothelin levels in Kawasaki disease: a possible marker for Kawasaki disease, Angiology 44, 719 723, 1993.

27. S. Akerstrom, V. Gunalan, C. T. Keng, Y-J. Tan, and A. Mirazimi, Dual effect of nitric oxide on SARS-CoV replication: viral RNA production and palmitoylation of the S protein are affected, Virology 395, 1-9, 2009.

28. C. Kunsch, and R. M. Medford, Oxidative stress as a regulator of gene expression in the vasculature, Circ. Res. 85, 753-766, 1999.

29. M. Merad, and J. C. Martin, Pathological inflammation in patients with COVID-19: a key role for monocytes and macrophages, Nat. Rev. Immunol. 20, 355-362, 2020.

30. S. Chen, B. Feng, B. George, R. Chakrabarti, M. Chen, and S. Chakrabarti, Transcriptional coactivator p300 regulates glucose induced gene expression in the endothelial cells, Am. J. Physiol. Endocrinol. Metab. 298, E127-E137, 2010.

31. J. Aitsebaoma, M. L. Kingsley-Kallesen, Y. Wu, T. Quertermous, and C. Patterson, Vezf1/DB1 is an endothelial cell-specific transcriptional factor that regulates expression of the endothelin-1 promoter, J. Biol. Chem. 276, 39197-39205, 2001.

32. J. Aitsebaomo, K. Wennerberg, C. J. Der, C. Zhang, V. Kedar, M. Moser, M. L. Kingsley-Kallesen, G. Q. Zeng, and C. Patterson, p68RacGAP is a novel GTPase-activating protein that interacts with vascular endothelial zinc factor-1 and modulates endothelial cell capillary formation, J. Biol. Chem. 279, 17963-17972, 2004.

33. Z. Yang, and J. C. Li, Stimulation of endothelin-1 gene expression by insulin via phosphoinositide3 kinase-glycogen synthase kinase-3 $\beta$ signalling in endothelial cells, Life Sci. 82, 512-518, 2008.

34. F. Rubino, S. A. Amiel, P. Zimmet, G. Alberti, S. Bornstein, R. H. Eckel, G. Mingrone, B. Boehm, M. E. Cooper, Z. Chai, et al., New-onset diabetes in Covid-19, N. Engl. J. Med. doi: 101056/NEJMc2018688, 2020. 
35. M. Foschi, A. Sorokin, P. Pratt, A. McGinty, G. La Villa, F. Franchi, and M. J. Dunn, PreproEndotheli-1 expression in human mesangial cells: evidence for a p38 mitogen-activated protein kinase/protein kinases-C-dependent mechanism, J. Am. Soc. Nephrol. 12, 1137-1150, 2001.

36. T. Imai, Y. Hirata, T. Emori, M. Yanagisawa, T. Masaki, and F. Marumo, Induction of endothelin1 gene by angiotensin and vasopressin in endothelial cells, Hypertension 19, 753-757, 1992.

37. M. Vaduganathan, O. Vardeny, T. Michel, J. J. V. McMurray, M. A. Pfeffer, and S. D. Solomon, Renin-angiotensin-aldosterone system inhibitors in patients with Covid-19, N. Engl. J. Med. 382, 1653-1659, 2020.

38. Y. Liu, Y. Yang, C. Zhang, F. Huang, F. Wang, J. Yuan, Z. Wang, J. Li, J. Li, C. Feng, et al., Clinical and biochemical indexes from 2019-nCoV infected patients linked to viral loads and lung injury, Sci. China Life Sci. 63, 364-374, 2020.

39. K. Yamashita, D. J. Discher, J. Hu, N. H. Bishopric, and K. A. Webster, Molecular regulation of the endothelin-1 gene by hypoxia. Contributions of hypoxia-inducible factor-1, activator protein-1, GATA-2, and p300/CBP, J. Biol. Chem. 276, 12645-12653, 2001.

40. G. P. Rossi, T. M. Seccia, G. Albertin, and A. C. Pessina, Measurement of endothelin: clinical and research use, Ann. Clin. Biochem. 37, 608-626, 2000.

41. B. Zhang, J. Price, G. Hong, S. M. Tabakman, H. Wang, J. A. Jarrell, J. Feng, P. J. Utz, and H. Dai, Multiplexed cytokine detection on plasmonic gold substrates with enhanced near-infrared fluorescence, Nano Res. 6, 113-120, 2013.

42. H. Hakonarson, E. Halapi, R. Whelan, J. Gulcher, K. Stefansson, and M. M. Grunstein, Association between IL-1 $\beta /$ TNF- $\alpha$-induced glucocorticoid-sensitive changes in multiple gene expression and altered responsiveness in airway smooth muscle, Am. J. Respir. Cell Mol. Biol. 25, 761-771, 2001.

43. J-M. Berthelot, and F. Lioté, COVID-19 as a STING disorder with delayed over-secretion of interferon-beta, EBioMedicine 56, 102801, 2020. 